\title{
Some effects of distance and structure on conjunction errors
}

\author{
JACK L. GALLANT and W. R. GARNER \\ Yale University, New Haven, Connecticut
}

\begin{abstract}
Conjunction errors may occur when location information is lost or degraded, so that stimulus attributes that are physically separate in a display appear to be conjoined. Structural influences on conjunction error rates have been studied extensively, but distance effects obtained over different experiments have been inconsistent. These effects may be an important source of information concerning the nature of conjunction errors. In this experiment, the proximity of stimulus items was varied within two structurally different stimulus sets. Both sets produced high conjunction error rates for adjacent items, whereas rates differed for the two sets over greater distances.
\end{abstract}

When the difficulty of a visual search task is increased by presenting the search field only briefly and following it with a pattern mask, subjects often respond to distractors as if they were targets. This pattern of errors occurs whenever the target's attributes exist across the set of distractors, even though no individual distractors share all of the attributes of the target. These particular errors have come to be known as "illusory conjunctions" (Treisman, Sykes, \& Gelade, 1978). For example, if an observer were required to search for a cross in a field of vertical and horizontal lines, he/she would occasionally respond that a cross was present when the field contained only vertical and horizontal lines. If the number of errors made when only verticals and horizontals are present significantly exceeds the errors made when only verticals are present, the excess is taken to reflect the rate of illusory conjunctions (Prinzmetal, 1981; Treisman \& Paterson, 1984). Because illusory conjunctions are inferred on the basis of error rates rather than on the basis of subjective experience, we use the operationally preferable term conjunction errors.

\section{Evidence for Conjunction Errors}

Conjunction errors that involve the dimensions of color and form have been studied most extensively, and occur under widely varying display and response conditions. When a field of colored letters is presented briefly and backward masked, subjects make more errors to displays separately containing the letter and the color than would be expected by chance (Treisman \& Schmidt, 1982) or to displays containing the letter or color attributes alone (Treisman et al., 1978). When colored or uncolored letters in a display form words, conjunction errors are more likely to occur within words or syllables than between them (Mozer, 1983; Prinzmetal \& Millis-Wright, 1984).

This research was supported by Grant MH 14229 from the National Institute of Mental Health to Yale University. We thank Arthur Samuel for valuable comments on an earlier version of this paper. Requests for reprints should be sent to either J. L. Gallant or W. R. Garner, Department of Psychology, Box 11-A Yale Station, New Haven, CT 06520.
Structural effects. Conjunction errors of color and shape occur if individual distractors share the shape of the target but differ in color. If distractors differ only in shape, the errors can occur even if the target can be reconstructed only from parts of different distractors (Treisman et al., 1978). The shapes of distractors have complex effects upon conjunction error rates. When the conjoining attributes are part of a distractor that is itself a "good" stimulus, conjunction errors that involve those attributes and attributes of other items in the stimulus field are less likely to occur than if the attributes are not part of a "good" stimulus (Treisman \& Paterson, 1984). If the search field is constructed so that targets and distractors are embedded within larger gestalt groups, conjunction errors that involve attributes from distractors in different groups are less likely to occur than errors that involve attributes from items in the same group (Prinzmetal, 1981).

Distance effects. The conjunction error literature is marked by a conspicuous absence of consistent distance effects. There have been some indications that conjunction error rates decrease as the attributes are separated by greater distances (Mozer, 1983; Prinzmetal \& MillisWright, 1984). On the other hand, some researchers have found only an unreliable adjacency effect upon the conjunction error rate (Treisman \& Schmidt, 1982). The lack of reliable distance effects may be due to the fact that studies of conjunction errors have not been explicitly designed to evaluate distance effects, and so the effects that have been found could not be confirmed with confidence.

\section{Necessary Conditions}

Although conjunction errors occur under a wide range of structural and display conditions, there are three broad constraints that must be satisfied in order for them to appear, although only the first of these has been studied systematically.

Shared attributes. In order for conjunction errors to be produced, a set of distractors must individually share some of the attributes of the target and collectively share all of the attributes. The target need not be specified be- 
forehand; conjunction errors may occur in free report tasks (Treisman \& Schmidt, 1982). Treisman and her coworkers (e.g., Treisman \& Gelade, 1980) claimed that conjunction errors will occur only between separable attributes, that is, attributes that can be selectively attended to in a classification task (Garner, 1974).

Positional uncertainty. Conjunction error tasks involve a degree of positional uncertainty, usually induced by randomizing the location and occurrence of the target, or by varying the position of distractors within the field.

Degraded stimulus. Conjunction errors occur only if the observer has a limited period of time in which to view the stimulus. This limitation is almost always imposed by backward masking the stimulus field, although it may also be achieved by showing displays in series (Intraub, 1985).

\section{The Present Experiment}

The occurrence of distance effects is the least consistent finding in the conjunction error literature. Treisman's feature integration theory (Treisman et al., 1978) denies the existence of distance effects and predicts a global loss of complex spatial information. Prinzmetal's (1981; Prinzmetal \& Millis-Wright, 1984) account of conjunction errors allows for distance effects, but makes no predictions concerning the pattern of effects, since they are determined by an unspecified perceptual parsing mechanism. Both accounts are based on the assumption that conjunction errors are due to a relatively high-level perceptual failure, the former in attention and the latter in parsing. Since distance effects are often found in sensory and perceptual tasks, we suspect that they may be present in the conjunction error task as well. Therefore, this study was explicitly designed to make any such effects apparent.

Three factors were manipulated in the experiment reported here: the structure of the targets and distractors, the presence of targets and distractors across displays, and the distance between items that might contribute to conjunction errors. Two stimulus sets that differed in structure were used. In the closed set, the target and the horizontal and vertical distractors were closed circles containing parallel horizontal and vertical lines (see Method). In the open set, the target and the horizontal and vertical distractors were broken circles containing similar parallel lines. In the closed set, the interior lines appeared to be contained within the figures, whereas in the open set, the interior lines appeared to be part of the figures.

There were three target conditions: the target-present, target-absent, and conjunction conditions (see Prinzmetal, 1981; Treisman \& Schmidt, 1982). In the target-present condition, a single target and one critical distractor were present. In the target-absent condition, no target was present, and the attributes of any two distractors in the display could not be recombined to form the target. In the conjunction condition, no target was present, but the attributes of the target were contained within the two critical distractors. In this experiment, the distance between target attributes in the target-absent and conjunction con- ditions was controlled, so that distance effects could be examined systematically.

\section{METHOD}

\section{Subjects}

Eight undergraduate students at Yale University participated in the experiment for course credit. There were 3 females and 5 males, and all had normal or corrected-to-normal vision.

\section{Stimulus Sets}

Each display contained eight items arranged in a circle about the fixation point and separated by $45^{\circ}$ (see Figure 1). Two critical items in each display were drawn from the two sets of three stimuli shown in Figure 2. In the closed stimulus set, the circles contained two parallel vertical and two parallel horizontal lines with the intersecting center segments removed (a cross), or two parallel vertical lines, or two parallel horizontal lines. In the open stimulus set, the edges of the circle between the endpoints of each pair of lines were removed. For both stimulus sets, the circle containing a cross was the target, and those containing single pairs of vertical or horizontal lines were the distractors. In addition to the two critical items, each display contained six irrelevent continuous circles with no internal lines.

The distance from the fixation point to the nearest edge of each item was $1.05^{\circ}$, and the items themselves had an outside diameter of $0.7^{\circ}$. The entire display was $3.5^{\circ}$ across. The inside distance between the parallel lines was $0.1^{\circ}$. The stimuli were drawn in black ink on white cards, and the thickness of all lines was $0.03^{\circ}$.

\section{Target Conditions}

The critical items in each stimulus display were selected according to the target conditions as follows: Target-present: Target-present stimuli always contained one cross, and either one vertical or horizontal critical item, chosen randomly. Target-absent: Target-absent stimuli contained two vertical or two horizontal items, again chosen randomly. Conjunction: Conjunction stimuli always contained one horizontal and one vertical critical item.

\section{Distance}

In each target condition, the critical items appeared at all possible pairs of locations, producing 28 different displays per target condition and 84 displays per stimulus set. The distance between the critical items varied from one to four locations, as measured along the shortest path around the circle. All items in the displays were separated by $.875^{\circ}$ around the circumference, so the distances examined, measured from the centers of the critical items, varied from $.875^{\circ}$ to $3.5^{\circ}$ along the circumference.

\section{Stimulus Presentation}

The stimuli were presented briefly and were backward masked.

Mask. The stimulus masks were created by randomly placing black dots in approximately $40 \%$ of the locations in an imaginary grid of squares overlaying the mask card. The dots were each $0.075^{\circ}$ in diameter, and were separated from one another by a vertical and horizontal

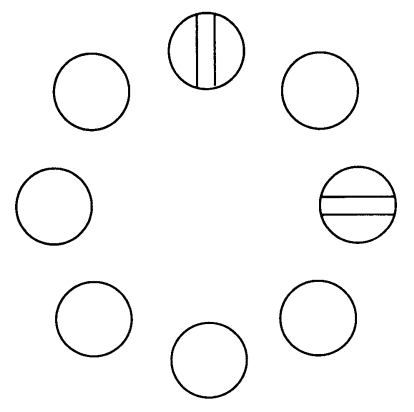

Figure 1. A conjunction error stimulus display from the closed stimulus set, with a distance of 2 . 


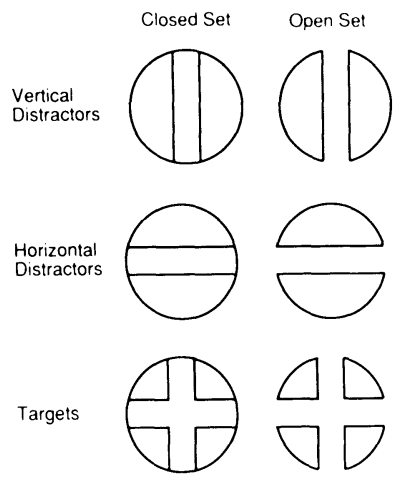

Figure 2. The complete set of stimuli, consisting of vertical distractors, horizontal distractors, and targets within closed and open stimulus sets.

distance of $0.025^{\circ}$. The mask field as a whole subtended $7.1^{\circ}$ horizontally and $4.9^{\circ}$ vertically. Two masking fields were constructed, differing only in the random placement of dots, and the masks were interchanged randomly throughout the experiment.

Practice and exposure duration. The subjects were induced to make errors $15 \%$ of the time in the target-present and target-absent conditions (averaged together), by manipulation of the stimulus duration. The experiment was run in blocks of $\mathbf{4 5}$ trials. After each block, the exposure duration was adjusted according to the following formula: If the average error rate in the target-present and target-absent conditions was between $10 \%$ and $20 \%$, the duration was not changed on that block. If it was between $5 \%$ and $10 \%$, the duration was decreased by $5 \mathrm{msec}$, and if it was less than $5 \%$ the decrease was $10 \mathrm{msec}$. If the average error rate was between $20 \%$ and $25 \%$, the duration was increased by $5 \mathrm{msec}$, and if it was over $25 \%$ the increase was $10 \mathrm{msec}$. If the exposure duration ever decreased below $35 \mathrm{msec}$, further decreases were always made in steps of only $5 \mathrm{msec}$. The mask was always shown for $500 \mathrm{msec}$.

Before data collection, the subjects performed several blocks of 10 practice trials each. The initial exposure duration was $50 \mathrm{msec}$, and was adjusted by an approximation of the experimental procedure after every block. The subjects practiced until they achieved between $80 \%$ and $90 \%$ correct responses on two successive practice blocks.

The overall mean exposure duration for the closed stimulus set was $46.0 \mathrm{msec}$, and for the open set was $49.6 \mathrm{msec}$. Individual subjects ranged from a mean duration of $34.4 \mathrm{msec}$ to $57.5 \mathrm{msec}$ with the closed set, and $26.9 \mathrm{msec}$ to $100 \mathrm{msec}$ with the open set. The difference in mean exposure durations across the two stimulus sets was not significant $[t(7)=-.043, p>.1]$. Each subject required shorter exposure durations on the second experimental session than the first to achieve the same average error rate.

\section{Procedure}

The experiment was run in two 1-h sessions, 1 to 4 days apart. In the first session, the subjects were given task instructions and were shown examples of some of the stimuli (two target-present, one target-absent, and one conjunction display). The subjects were told to try to be as fast and as accurate in their performance as possible. No mention was made of the exact ratio of targets to nontargets in the experiment (1:2). The sessions were blocked so that the subjects saw only one stimulus set each day, with the order of presentation of the sets counterbalanced across subjects. In each session, the subjects practiced until they reached the criterion error rate, and then ran through the set of 84 stimuli four times, for a total of 336 data trials. Two randomized card orders were used for each set, one for the first 4 and one for the second 4 subjects, but each repetition began at a different place in the order.

The experiment was run on a Scientific Prototype three-field tachistoscope. Trials were signaled by the sound of the stimulus card being inserted into the device. The subjects initiated stimulus presentation by fixating at the center of a blank field, and then pressing a button held in their nondominant hand. After a 500-msec delay, a stimulus field appeared for the prescribed duration, and was followed by the masking field. The subjects responded by pressing a switch up or down (for target present or absent) with their dominant hand, and the mapping of switch direction onto responses was counterbalanced across subjects. Both simple responses and reaction times were collected throughout the experiment, but since no significant effects were found in the reaction time data, only response accuracy data are reported here. During the procedure, the subjects were allowed to rest briefly after every 45 trials.

\section{RESULTS}

\section{Preliminary Analyses}

Conjunction errors are often defined as the difference between the average of the target-present and target-absent error rates, and the conjunction condition error rate (e.g., Prinzmetal, 1981; Treisman \& Paterson, 1984). However, we define the conjunction error rate as the simple difference between target-absent and conjunction condition error rates, because these two rates provide the critical comparison.

Before performance with the two stimulus sets could be compared, it was necessary to ensure that target-present and target-absent error rates were constant across stimulus sets. There were no significant differences in the target-present error rates found between the two sets (see Table 1). The target-absent error rates obtained with the two sets were identical, at $17 \%$. Therefore, the two stimulus sets contain equally discriminable targets.

The overall conjunction error data were analyzed using a stimulus set $(2) \times$ distance $(4)$ crossed repeated measures analysis of variance (ANOVA). The main effect of stimulus set was significant $(F=25.49, p<.01)$, whereas the main effect of distance barely failed to reach significance $(F=2.96, p=.056)$ and the interaction term was not significant. Although the interaction failed to reach significance by an omnibus $F$ test, we expected a priori that there may have been unique distance effects within each stimulus set. To examine these effects, an ANOVA was carried out independently on each stimulus set, and a set of orthogonal contrasts was performed across distance within each set.

\section{Closed Stimuli}

As shown in Table 1, the target-absent error rate was $17.0 \%$, and the conjunction condition error rate was $30.1 \%$. The overall conjunction error rate was therefore $13.2 \%$, which was significantly different from zero $[t(7)$ $=8.96, p<.001]$. This was a very robust finding; every subject showed the effect, with the conjunction error rate varying from $7.1 \%$ to $17.8 \%$. The conjunction error rates within each distance are also presented in Table 1 . The critical items in each display were separated by from one to four locations, so in order to determine the trend of conjunction error rates over distance, a set of orthogonal contrasts over distance was performed within a subjects $\times$ distance ANOVA for the closed data alone. The conjunction error rate obtained at the closest distance was compared with the average of the farther three distances, the next closest was compared with the average of the following two, and the farthest two distances were compared with each other. None of the con- 
Table 1

Error Rates (\%) for the Three Target Conditions and Conjunction Error Rates by Distance, for Closed and Open Stimulus Sets

\begin{tabular}{ccccr}
\hline & \multicolumn{3}{c}{ Target Conditions } & \\
\cline { 2 - 4 } Distance & $\begin{array}{c}\text { Target- } \\
\text { Present }\end{array}$ & $\begin{array}{c}\text { Target- } \\
\text { Absent }\end{array}$ & Conjunction & $\begin{array}{c}\text { Conjunction } \\
\text { Errors }\end{array}$ \\
\hline \multicolumn{4}{c}{ Closed Stimulus Set } \\
1 & 12.5 & 22.3 & 38.7 & \\
2 & 13.3 & 15.6 & 32.5 & 16.4 \\
3 & 11.0 & 14.8 & 22.7 & 16.9 \\
4 & 14.9 & 13.3 & 23.5 & 10.2 \\
Overall* & 12.6 & 17.0 & 30.1 & 13.2 \\
& & Open Stimulus Set & \\
1 & 13.7 & 14.5 & 29.3 & 14.8 \\
2 & 11.3 & 17.2 & 19.1 & 1.9 \\
3 & 16.4 & 16.8 & 13.7 & -3.1 \\
4 & 10.9 & 21.9 & 17.2 & -4.7 \\
Overall* & 13.4 & 17.0 & 20.2 & 3.2 \\
\hline
\end{tabular}

*Overall values are computed from a weighted average, since only half as many observations were made at distance 4 as at the other three distances.

trasts was significant, indicating that for the closed stimulus set, the conjunction error rate did not vary over distance. Furthermore, at each distance the conjunction error rate was significantly greater than zero [smallest $t(7)$ $=2.31, p=.054]$.

\section{Open Stimuli}

As shown in Table 1, with the open set the target-absent error rate was $17.0 \%$, and the conjunction condition error rate was $20.2 \%$. There was substantial variability in the error rates of individual subjects, and the overall conjunction error rate was not significantly different from zero $[t(7)=1.66, p>.1]$. However, in order to evaluate distance effects more completely, a set of orthogonal contrasts over distance like those conducted with the closed stimulus set was performed. The contrast for distance 1 versus distances 2,3 , and 4 was significant $[F(1,21)=11.57, p<.005]$, but none of the other contrasts was significant. Thus the conjunction error rate at the closest distance was significantly greater than the conjunction error rate at the farthest three distances. Furthermore, in confirmation of this difference, the conjunction error rate at distance 1 was significantly greater than zero $[t(7)=3.59, p<.01]$, but the conjunction error rates at the farthest three distances were not significantly different from zero [largest $t(7)=0.89, p>.1$ ]

\section{DISCUSSION}

Overall, the subjects made a significant number of conjunction errors with the closed stimulus set, but an insignificant number with the open stimulus set. Thus the conjunction error rate is affected by the structural relationship between the critical conjoining attributes and their local surrounding contexts. Qualitatively, critical attributes that are contained within a larger stimulus item are often subject to conjunction errors, whereas those that are part of their contextual surround are relatively immune to such errors. This simple result confirms and extends the past find- ings of structural effects on conjunction errors (Prinzmetal, 1981; Treisman \& Paterson, 1984), demonstrating that local stimulus structure may have a significant influence on the occurrence of conjunction errors.

However, when the critical items in a display were adjacent to one another, there was no difference in the conjunction error rates obtained with the two sets, even though there were significant differences at each of the farthest three distances. Although stimulus structure may have a large effect on conjunction errors in general, this effect is lost when critical items are adjacent. A phenomenal argument based on structural differences between the two sets may account for the overall effects we have observed, but such an explanation cannot account for the different distance effects we found with the two sets.

The finding that structural differences break down when conjoining items are adjacent suggests that a local process operates separately and in addition to the global process usually cited in conjunction error studies (Mozer, 1983; Prinzmetal, 1981; Treisman \& Gelade, 1980). Since conjunction errors occur under extremely limited viewing conditions produced by brief exposure durations and pattern masking, it is possible that the local loss of position information reflects absolute limits in the ability of subjects to encode fine spatial detail from the display. Such an encoding failure could take the form of a loss of spatial resolution, or a loss of phase information. If the limited viewing conditions caused a local, probabilistic loss of spatial resolution, then we would expect that subjects would make adjacency errors on a certain proportion of trials, in addition to any conjunction errors they might make.

If conjunction errors of shape are the ultimate result of a local loss of positional information, what can we make of the claim that attention mediates conjunction errors? It is well known that attending to a small location in a field of briefly presented items increases detection and identification accuracy at that location, and selective attention of this sort has been shown to reduce the occurrence of conjunction errors (Prinzmetal, Presti, \& Posner, 1986). If selective spatial attention can reduce conjunction errors by reducing the short-range loss of spatial resolution due to masking, perhaps attention is best characterized as a noise reduction system, which can increase the effective local resolution when the prevailing conditions make such information relatively difficult to extract. On the other hand, if the distance effects we have observed reflect the activity of higher cognitive, reconstructive processes, then attention might simply be a bias effect.

\section{REFERENCES}

GARNER, W. R. (1974). The processing of information and structure. Hillsdale, NJ: Erlbaum.

INTRAUB, H. (1985). Visual dissociation: An illusory conjunction of pictures and form. Journal of Experimental Psychology: Human Perception \& Performance, 11, 431-442.

MozER, M. C. (1983). Letter migration in word perception. Journal of Experimental Psychology: Human Perception \& Performance, 9 , 565-569.

Prinzmetal, W. (1981). Principles of feature integration in visual perception. Perception \& Psychophysics, 30, 330-340.

Prinzmetal, W., \& Millis-Wright, P. (1984). Cognitive and linguistic factors affect visual feature integration. Cognitive Psychology, 16, 305-340.

Prinzmetal, W., Presti, D. E., \& Posner, M. I. (1986). Does attention affect visual feature integration? Journal of Experimental Psychology: Human Perception \& Performance, 12, 361-369.

Treisman, A., \& Gelade, G. (1980). A feature integration theory of attention. Cognitive Psychology, 12, 97-136.

Treisman, A., \& Paterson, R. (1984). Emergent features, attention, and object perception. Journal of Experimental Psychology: Human Perception \& Performance, 10, 12-31.

Treisman, A., \& Schmidt, H. (1982). Illusory conjunctions in the perception of objects. Cognitive Psychology, 14, 107-141.

Treisman, A., Sykes, M., \& Gelade, G. (1978). Selective attention and stimulus integration. In S. Dornic (Ed.), Attention and performance VI. Hillsdale, NJ: Erlbaum.

(Manuscript received for publication January 19, 1988.) 